\title{
Avaliar: Sistema para Autoria e acompanhamento de recursos avaliativos
}

\author{
Alana Elias \\ UFAPE \\ Garanhuns - PE \\ alana.elias@ufrp \\ e.br
}

\author{
Igor Vanderlei \\ UFAPE \\ Garanhuns - PE \\ igor.vanderlei@ \\ ufrpe.br
}

\author{
Mariel Andrade \\ UFAPE \\ Garanhuns - PE \\ mariel.andrade \\ @ufrpe.br
}

\author{
Rodrigo \\ Gusmão \\ UFAPE \\ Garanhuns - PE \\ rodrigo.gusmao \\ @ ufrpe.br
}

\author{
Jean Teixeira \\ UFAPE \\ Garanhuns - PE \\ jean.teixeira@uf \\ rpe.br
}

\begin{abstract}
Learning verification is the target of numerous studies that aim to transform it from its solely classificatory character, in a process of diagnosing the student's evolution in the learning goals of a subject. This research proposes a software for monitoring and authoring evaluative activities, modelled in order to support teachers and students in the learning verification process. For this, using the DSR methodology, a bibliographic study was carried out in the context of school evaluation to support the designing of the tool. The presented solution was validated through tests and questionnaires on user experience. The results demonstrated the potential of the tool to support the school evaluation process.
\end{abstract}

\section{KEYWORDS}

School Evaluation, Educational Softwares, Learning Management Systems

\section{Introdução}

A inserção das Tecnologias de Informação e Comunicação (TICs) no ambiente educacional abre espaço para a criação de novas práticas educativas, impulsionando a criação de diversos sistemas com o intuito de auxiliar professores e alunos no processo de ensino. Dentre estes, destacam-se os sistemas de autoria, descritos como programas de computador utilizados para a produção de arquivos digitais. Os sistemas de autoria objetivam proporcionar aos docentes uma plataforma para construir, de forma automatizada e simples, recursos educacionais digitais. (Leffa, 2006)

Na mesma categoria de ferramentas de apoio à docência, os Sistemas de Gestão de Aprendizagem (SGA) são definidos como portais online que conectam professores e alunos, oferecem uma maneira fácil de compartilhar materiais, concentrando um conjunto de funcionalidades, para criar e gerir um espaço de monitoramento e avaliação com a finalidade de apoiar o processo de aprendizado (Adzharuddin e Ling, 2013).

Diversas áreas da educação podem beneficiar-se da inclusão de

In: XIX Workshop de Ferramentas e Aplicações (WFA2020), São Luís, Brasil. Anais Estendidos do Simpósio Brasileiro de Sistemas Multimídia e Web (WebMedia). Porto Alegre: Sociedade Brasileira de Computação, 2020.

(C)2020 SBC - Sociedade Brasileira de Computação.

ISSN: 2596-1683 novas tecnologias, dentre elas a avaliação de aprendizagem. O tema 'avaliação da aprendizagem escolar' aparece como um dos grandes desafios do sistema educacional (Marcante, Darroz e Rosa, 2012). Os modelos avaliativos procuram cada vez mais basear-se no acompanhamento do desempenho individual dos estudantes nas diversas atividades realizadas no decorrer da disciplina, complementando modelos mais antigos que se utilizavam apenas de um tipo de avaliação. Essa percepção, no entanto, suscita dificuldades no acompanhamento do processo de verificação de aprendizagem em sala de aula, levando grande parte dos docentes a permanecerem utilizando avaliação de caráter classificatório.

Diante das dificuldades presentes no processo de verificação de aprendizagem e os potenciais benefícios da inclusão das TIC's no âmbito educacional, o presente trabalho visa apresentar um sistema open-source que combina conceitos das ferramentas de autoria e dos SGA em um único ambiente. O software objetiva apoiar os docentes na avaliação de aprendizagem, contribuindo com o acompanhamento do desenvolvimento dos alunos. $\mathrm{O}$ sistema pode ser adotado por docentes e escolas de diversos níveis de ensino, auxiliando professores que busquem diferentes maneiras de lidar com o processo avaliativo.

O restante do artigo está organizado da seguinte maneira: A Seção 2 detalha fundamentação teórica utilizada como base do sistema proposto, a Seção 3 descreve a metodologia utilizada neste trabalho; na Seção 4 é apresentado o sistema proposto e suas funcionalidades; a Seção 5 apresenta a discussão dos resultados; seção 6 apresenta sistemas semelhantes, por fim, a Seção 7 apresenta as conclusões da pesquisa e sugestões de trabalhos futuros.

\section{Fundamentação teórica}

Esta seção apresenta o estudo bibliográfico que fundamentou a modelagem e construção do software proposto. O principal foco foi o estudo da inclusão das TIC's na educação, analisando o cenário da avaliação de aprendizagem escolar e o potencial impacto de SGAs e sistemas de autoria neste âmbito.

\subsection{Avaliação de aprendizagem escolar}

As concepções de ensino e aprendizagem estão em constante evolução. Vieira (2002) defende que as mudanças ocorridas nesse âmbito resultam em repercussões importantes no campo das práticas das avaliações escolares. A avaliação torna-se um 
instrumento fundamental para repensar e reformular os métodos e estratégias de ensino para que, de fato, o aluno aprenda. Esta deve ser entendida pelo professor como processo de acompanhamento e compreensão dos avanços, dos limites e das dificuldades dos alunos a fim de atingir os objetivos da atividade de que participam (Duarte, 2015). Diante disso, o professor como avaliador tem o papel de coletar essas informações, a fim de acompanhar o avanço individual de seus discentes, no intuito de guiá-los a atingir os objetivos esperados.

Essa perspectiva deixa de considerar a avaliação apenas como uma classificação (aprovado ou reprovado), e passa a caracterizála como uma maneira de identificar e obter informações relevantes sobre o processo ensino-aprendizagem. Para Luckesi (2005), a avaliação com a função apenas classificatória, constituise num instrumento estático dificultando o processo de crescimento.

Luckesi (2005) define ainda que a avaliação deverá ser assumida como um instrumento de compreensão do estágio de aprendizagem em que se encontra o aluno, com o objetivo de tomar decisões suficientes e satisfatórias para que o mesmo possa avançar no seu processo de aprendizagem. Nesta perspectiva, destaca-se a importância de prover ao docente todas as informações necessárias no que diz respeito ao acompanhamento da avaliação, tornando-a uma ferramenta essencial ao processo de aprendizagem.

\subsection{Sistemas de gestão de aprendizagem}

A busca dos profissionais de educação em superar as dificuldades no que concerne a sua prática, especialmente no que diz respeito à sala de aula, inclui a inserção da tecnologia às práticas educacionais (Luis e Silva, 2008). Neste cenário, surgem diversas categorias de sistemas de apoio à docência, dentre eles, destacamse os Sistemas de Gestão da Aprendizagem (SGA), que compreendem funcionalidades definidas para armazenar, distribuir e gerenciar conteúdos de aprendizado de forma interativa e gradativa (Carvalho et al., 2012).

Ayubet al. (2010) definem SGA como uma tecnologia baseada na web que auxilia no planejamento, distribuição e avaliação de um processo de aprendizagem específico. Gomes et al. (2009) defendem que os SGAs são ferramentas imprescindíveis ao processo de ensino/aprendizagem, que permitem acompanhar a construção do conhecimento individual dos alunos por meio do registro da discussão, reflexão e colaboração.

Essas plataformas possuem como objetivo principal proporcionar um ambiente virtual para apoiar o processo de acompanhamento entre o professor e seus alunos, facilitando a interação e assistência. Por sua característica de prover um ambiente educacional de maneira virtual essa ferramenta se popularizou na esfera do ensino a distância.

\subsection{Sistemas de autoria}

Ainda no cenário de inclusão de TICs no ambiente educacional, ressalta-se a relevância dos sistemas de autoria, que provêm um ambiente de simples uso para a criação de recursos educacionais digitais. Os softwares de autoria são utilizados para desenvolver aplicações multimídias em diversas áreas. $\mathrm{Na}$ educação, os sistemas de autoria permitem que o professor e os alunos criem, implementem e testem aplicações (Falkembach, 2007). Por oferecer facilidade na elaboração de diversos tipos de objetos multimídia, esse tipo de sistema pode se tornar um aliado no processo de avaliação, permitindo que o professor utilize seus recursos para criar atividades e listas avaliativas.

No contexto de verificação de aprendizagem escolar, os sistemas de autoria possuem uma importante contribuição. Através de sua característica de permitir a criação de objetos de aprendizagem, tornam-se poderosas ferramentas para a elaboração de atividades avaliativas de conteúdos diversos. Além dos ambientes já existentes de EAD, que proporcionam a criação de avaliações com inúmeros benefícios, existem também ferramentas de autoria multimídia, que também implementam diversas características inerentes à avaliação e possuem, cada vez mais, recursos de disponibilização de seus aplicativos na Web (Cardoso, 2001).

\section{Metodologia}

O desenvolvimento desta pesquisa foi estruturado utilizando a abordagem Design Science Research (DSR), caracterizada por elencar dois objetivos principais, centrados em: i) resolução de um problema através da inserção de um artefato proposto; e ii) gerar novo conhecimento científico (Pimentel, Filippo e Santoro, 2019).

O presente trabalho situou-se na problemática da avaliação da aprendizagem escolar e no estudo de suas dificuldades, culminando no problema de pesquisa. Partindo da premissa que as tecnologias podem contribuir com a educação, foi proposto um artefato, um sistema de gestão de aprendizagem e autoria de recursos educacionais, como um instrumento de apoio ao acompanhamento e verificação de aprendizagem.

Esta pesquisa iniciou-se com a investigação do problema, que consistiu na realização de uma análise acerca das principais dificuldades no contexto da verificação de aprendizagem escolar. A partir da base adquirida, foram identificadas funcionalidades importantes para a finalidade do sistema proposto, culminando em um conjunto de histórias de usuário, com objetivo de nortear a construção do software.

A solução proposta foi implementada utilizando a metodologia Scrum, uma metodologia ágil com foco em sua característica incremental que oferece uma maior adaptabilidade aos requisitos passíveis de modificações e prazos curtos (Sommerville, 2011).

Após a finalização da versão inicial do sistema, foi realizada a validação, que contou com a seleção de potenciais usuários para utilização do sistema desenvolvido, realizando uma pesquisa com a participação de alunos de uma turma de alunos e professores de um curso de Licenciatura em Pedagogia.

\section{Sistema Proposto}

O sistema Avaliar tem por objetivo facilitar o processo de verificação de aprendizagem de quaisquer disciplinas e níveis de ensino. Para tanto, conta com funcionalidades de gestão de aprendizagem e de autoria de recursos que permitem a criação de listas de atividades sobre tópicos específicos de uma disciplina, e o acompanhamento individual dos resultados. O sistema pode ser acessado através do link http://app.uag.ufrpe.br/avaliar/, e seu código-fonte está disponível em https://github.com/AlanaTenorio/SistemaAvaliacao.

\subsection{Arquitetura do Sistema}


Avaliar: Sistema para autoria e acompanhamento de recursos avaliativos

A arquitetura definida para o software é apresentada na Figura 1. $\mathrm{O}$ acesso ao sistema é realizado via $W e b$, através de requisições $h t t p$, interagindo com o código implementado na linguagem $P H P$, utilizando-se da ferramenta Laravel, que armazena os dados em um banco de dados PostgreSQL.

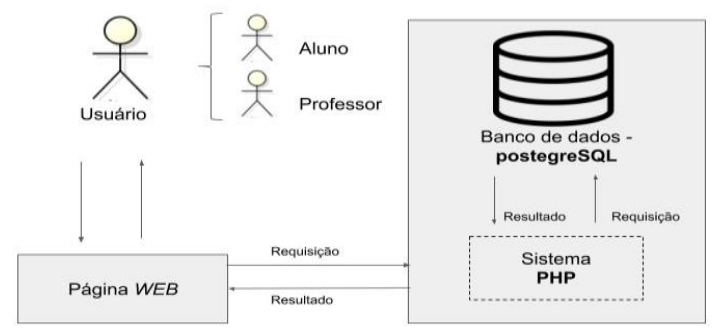

Figura 1: Arquitetura do sistema

O software foi desenvolvido utilizando a linguagem de programação $P H P$, com o framework Laravel e incorporando a arquitetura $M V C$, padrão de arquitetura de software que divide o projeto em três camadas principais. Os tópicos a seguir descrevem os módulos do sistema e suas principais funcionalidades.

\subsection{Principais Funcionalidades}

Os tópicos a seguir detalham as principais funcionalidades do sistema proposto.

\subsubsection{Módulo Professor}

O docente, como usuário do sistema, possui acesso às funcionalidades de gestão e autoria de recursos educacionais. $\mathrm{O}$ módulo de gestão permite o cadastro de uma turma através de um formulário solicitando as informações da mesma. Após a criação de uma turma, o professor autor poderá inserir seus conteúdos, compartilhá-la por e-mail, para que seus alunos obtenham acesso aos conteúdos publicados na turma e visualizar todos os alunos participantes, assim como funções de gerenciamento básicas.

Com o objetivo de facilitar o mapeamento dos resultados dos alunos nas listas de atividades, é gerado um mapa de conteúdos a partir dos conteúdos inseridos pelo professor em uma turma, relacionando todas as competências da disciplina e suas respectivas dependências. A Figura 2 mostra um exemplo de mapeamento de conteúdos.

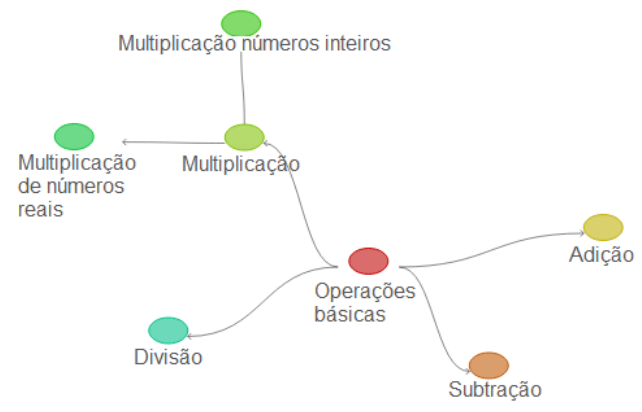

Figura 2: Mapa de conteúdos
Anais Estendidos do WebMedia'2020, São Luís, Brasil

O módulo de Autoria de recursos possibilita a criação de atividades e listas de exercícios a serem respondidas pelo aluno, que serão corrigidas automaticamente pelo sistema, a partir das respostas incluídas pelo professor na criação da lista, e os resultados dos alunos serão exibidos para o professor. Atualmente o sistema suporta três tipos de atividades: i) Múltipla-escolha; ii) Associação de imagem-texto; iii) Associação de imagem-áudio. A Figura 3 exemplifica a criação de uma atividade do tipo MúltiplaEscolha.

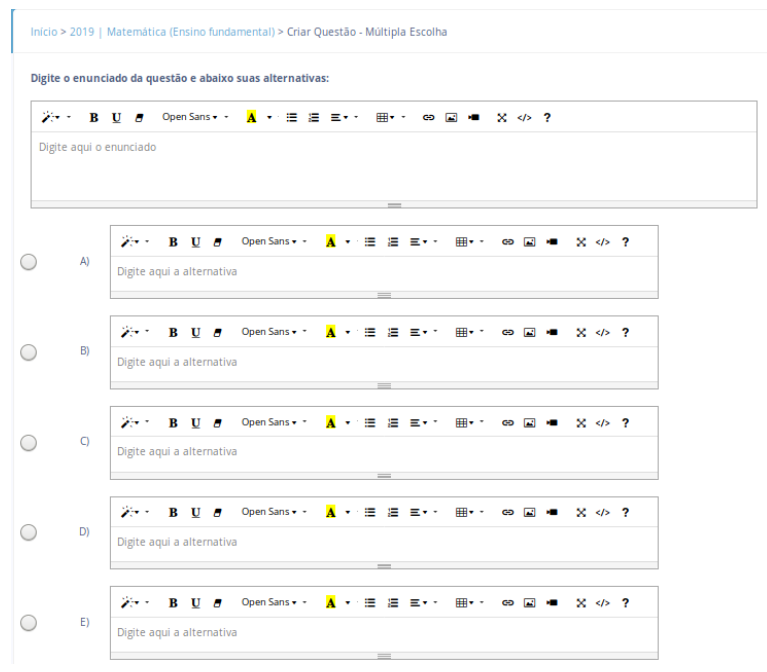

Figura 3: Atividade de múltipla-escolha

Cada atividade está associada a um conteúdo da Turma, os resultados dos alunos são expostos de maneira a permitir o acompanhamento de seu desempenho nos conteúdos específicos da disciplina. A Figura 4 mostra os resultados dos alunos em uma lista de atividades.

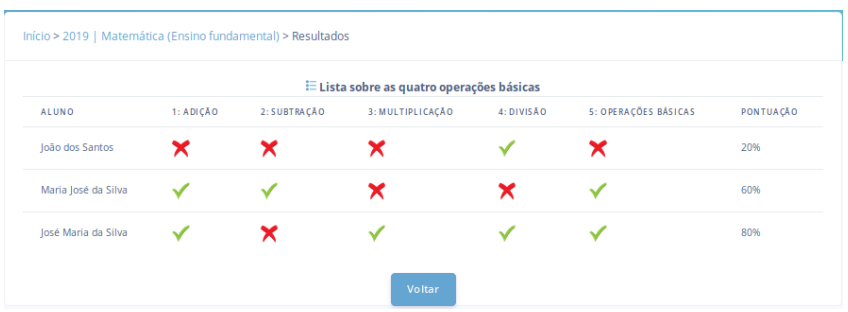

Figura 4: Resultados de uma lista

\subsubsection{Módulo Aluno}

O usuário do tipo Aluno possui funcionalidades que dizem respeito à sua participação em determinada turma: responder atividades organizadas em listas, e acompanhar seu desenvolvimento nos conteúdos da mesma. Após a publicação de uma lista de atividades para uma turma, esta estará disponível para ser respondida pelo aluno que participa dela, que pode acessá-la através da sua listagem de atividades avaliativas ainda não respondidas. Todas as questões inseridas na lista são apresentadas ao usuário, que pode respondê-las até a data final 
definida pelo professor no momento de criação. A Figura 5 mostra como o aluno pode responder uma atividade do tipo Associação de Imagem-texto.

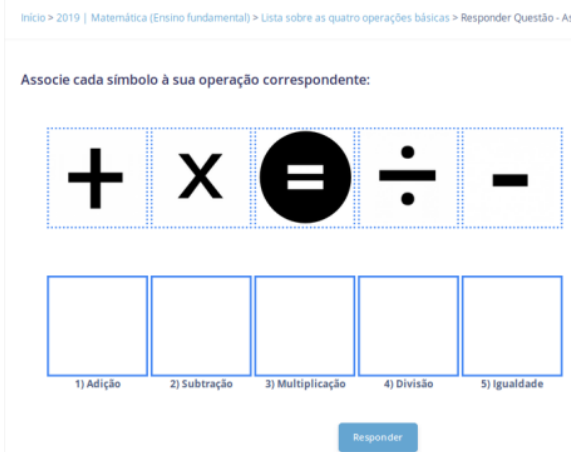

Figura 5: Atividade de associação imagem-texto

Após a finalização de uma lista, o aluno pode verificar seus resultados, a partir de uma tabela que lista as questões respondidas ou não corretamente, e seu respectivo assunto. Esta visualização permite que o aluno possua um autoacompanhamento de seu desempenho, observando seus assuntos de maiores dificuldades.

\section{Resultados e discussão}

Esta etapa possuiu como objetivo analisar a usabilidade geral do sistema, bem como o impacto de sua utilização, visando observar as principais dificuldades dos usuários na utilização do mesmo, perceber a necessidade de novas funcionalidades, e obter uma visão geral do público-alvo em relação ao sistema.

A pesquisa foi realizada em uma turma de 26 alunos, e 2 professores de um curso de Licenciatura em Pedagogia. Com o intuito de analisar os módulos acessados tanto pelo Professor quanto pelo Aluno, a turma foi dividida para realizar os testes em ambos os módulos: 17 usuários desempenharam papel de professor, e 9 desempenharam papel de aluno. Para melhor analisar os dados obtidos, a pesquisa foi dividida em duas seções.

\subsection{Avaliação da pesquisa}

Essa fase buscou validar o potencial do sistema em contribuir com o processo de verificação de aprendizagem, ajudando a solucionar as principais dificuldades citadas neste trabalho. A análise foi conduzida monitorando os usuários de teste durante a realização de operações no sistema, solicitadas através de um roteiro de testes. Esta etapa focou em observar os possíveis benefícios do sistema para o processo avaliativo.

Após o uso do sistema, os usuários foram questionados em relação aos recursos disponíveis para a criação de listas de atividades que compõem o módulo de autoria do sistema. Obtendo como resultado que o modelo atual de criação de listas atende às principais necessidades do professor, e como sugestão a inclusão de outros tipos de atividades na composição da lista.

Em relação à visualização dos resultados das atividades, os participantes ressaltaram que o modelo de mapeamento de conteúdos proposto pelo sistema facilita tanto no planejamento geral da disciplina, como na exibição dos resultados dos alunos, permitindo uma visão mais clara do acompanhamento do nível de conhecimento da turma em cada conteúdo, tanto para o aluno quanto para o professor, potencialmente contribuindo para a análise das principais dificuldades de cada aluno em cada competência da disciplina.

Foi ressaltado ainda, que por combinar funcionalidades de autoria com as funcionalidades de gestão de turmas comuns a outros sistemas educacionais, o Avaliar tem o potencial de causar impactos positivos, permitindo diagnosticar mais cedo as dificuldades dos estudantes em relação ao conteúdo da turma. Além disso, foi destacado o benefício do sistema para o estudo individual do aluno, que possui acesso ao mapeamento dos seus resultados, identificando suas áreas de maior dificuldade.

Por fim, os usuários foram questionados quanto ao potencial geral da ferramenta no apoio ao processo de avaliação escolar. Todos os usuários participantes mencionaram que a ferramenta tem o potencial de auxiliar na verificação de aprendizagem escolar, servindo de instrumento para acompanhar o desempenho do aluno.

Diante dos resultados apresentados, é possível observar que, de acordo com a percepção dos participantes no processo de avaliação, o uso de uma ferramenta de gestão e autoria de recursos avaliativos tem o potencial de auxiliar no processo de verificação de aprendizagem. A visualização dos resultados por meio de conteúdos permite uma visão mais clara do desenvolvimento dos alunos em cada competência, facilitando o acompanhamento das principais dificuldades.

\subsection{Avaliação do artefato}

Nesta etapa foi analisada a usabilidade geral do sistema, visando observar as principais dificuldades dos usuários na utilização do mesmo, perceber a necessidade de novas funcionalidades, assim como obter uma visão geral do público-alvo em relação ao sistema.

A pesquisa foi conduzida de maneira similar à validação da pesquisa, desta vez com o foco na experiência de uso geral do sistema, a fim de mapear possíveis problemas de usabilidade e obter sugestões de potenciais melhorias.

Os resultados obtidos indicaram que $88 \%$ dos usuários não relataram dificuldades ao realizar as operações no sistema propostas pelo questionário. Quando questionados sobre possíveis funcionalidades futuras, os usuários ressaltaram a possibilidade de um ambiente de interação entre professores e alunos, possibilitando comentários e sugestões.

Diante desses resultados, foi observado que a usabilidade do sistema no geral apresentou poucas dificuldades, a maioria são centradas no módulo de Professor, o que forneceu informações importantes de melhorias e novas funcionalidades que poderão ser implementadas em versões futuras do sistema.

\section{Trabalhos relacionados}

Esta seção analisa sistemas educacionais livres encontrados, observando seus principais módulos e funcionalidades, com foco em Sistemas de Gestão de Aprendizagem e ferramentas de autoria.

\subsection{Ardora}


Avaliar: Sistema para autoria e acompanhamento de recursos avaliativos

O Ardora (ARDORA, 2009) define-se como uma ferramenta livre, que permite a criação de atividades de diversos tipos: quebra cabeça, associação entre imagem e texto, associação entre som e imagem, palavras-cruzadas, dentre outras. Sua interface permite a inserção tanto de objetos textuais como multimídia (imagens, áudios, vídeos). A proposta principal desse sistema de autoria é a de facilitar criação de objetos virtuais de aprendizagem, permitindo a elaboração de atividades para todos os níveis de ensino.

A análise do Adora permitiu observar que o sistema é bastante completo em relação às ferramentas de autoria, possibilitando a criação de diversos tipos de atividades, dentre elas associação de imagem-texto, associação de imagem-áudio, caça -palavras, entre outros. O sistema, no entanto, não disponibiliza funcionalidades de gestão e acompanhamento das respostas dos alunos.

\subsection{Khan Academy}

Khan Academy é uma plataforma online que se define como "uma organização sem fins lucrativos com o objetivo de mudar a educação para melhor, oferecendo uma educação gratuita de classe mundial para qualquer pessoa em qualquer lugar". O site provê um ambiente de aprendizado com vídeo-aulas e mecanismos de geração de problemas baseando-se no nível atual de habilidade do aluno.

Uma das importantes contribuições do Khan Academy é a possibilidade de aluno e professor acompanhar o desenvolvimento do conhecimento em tempo real através da plataforma. O sistema disponibiliza uma grande variedade de tutoriais e questões, bem como ferramentas precisas de acompanhamento para professores e tutores, porém sua versão atual não permite a criação inserção de novas atividades ou listas, limitando-se apenas as que já estão previamente inseridas.

\section{Conclusão}

Diversos estudos no contexto de avaliação escolar referem-se aos problemas encontrados no processo avaliativo. O caráter apenas classificatório por vezes não se mostra eficiente para uma ampla observação das principais dificuldades e evolução de um aluno. Neste ponto, a inserção das TICs na educação pode se tornar um forte aliado no auxílio à prática docente, com sistemas capazes de gerar benefícios para professores e alunos no processo de ensinoaprendizagem. Diante deste problema, foi proposto um sistema open-source de autoria e acompanhamento de recursos avaliativos, permitindo ao professor uma visualização mais completa da evolução de seus alunos em relação a cada conteúdo da disciplina.

A pesquisa objetivou o desenvolvimento e avaliação da ferramenta, para tanto foi realizado um estudo bibliográfico em relação ao cenário da verificação de aprendizagem, analisando também o impacto da inclusão da tecnologia no ensino. O sistema foi modelado a partir deste estudo, culminando em um conjunto de requisitos, que orientou a produção e avaliação do artefato proposto.

A validação feita com potenciais usuários permitiu observar o potencial da ferramenta de auxiliar na verificação de
Anais Estendidos do WebMedia’2020, São Luís, Brasil

aprendizagem, trazendo ao professor e ao aluno um acompanhamento mais preciso dos conteúdos os quais os alunos apresentam maiores dificuldades e proporcionando um ambiente para facilitar e apoiar o processo de avaliação de aprendizagem escolar.

A partir dos resultados observados foi possível identificar potenciais trabalhos futuros como a realização de testes mais aprofundados com maior número de usuários, e o desenvolvimento de versões seguintes do sistema, como a inclusão de mais categorias para melhor atender as necessidades do professor e a inclusão de um ambiente interativo que proporcione a comunicação entre os estudantes e os docentes, com o objetivo de compartilhar conteúdos e possíveis dificuldades.

\section{REFERÊNCIAS}

[1] Adzharuddin N.A. e Ling, L.H. (2013) "Learning Management System (LMS) among Universuty Students: Does it Work?", International Journal of eEducation, e- Business, e-Management and E-learning, 3, páginas 248-252.

[2] Ayub A. F. M., Tarzimi, R. A, Jaafar, W. M. W, Ali, W. Z. W e Luan, W. S. (2010) 'Factors influencing students' use a Learning Management System Portal: Perspective from Higher Education Students", Int. J. Educ. Inf. Technol., v. 4, n. 2, p. 100-108.

[3] Cardoso, R. F. (2001) "AvalWeb - Sistema interativo para gerência de questões e aplicação de avaliações na Web.”, Universidade Federal do Rio Grande do Sul, Instituto de Informática.

[4] Carvalho, R. S., Filho, I. J. D. M., Vidal, T. C., Melo, R. M. e Gomes, A. S (2012) "Integração entre o sistema de gestão acadêmica e o sistema de gestão da aprendizagem: identificando necessidades e prototipando requisitos favoráveis a prática docente.”, Rev. Bras. Comput. Apl., v. 4, n. 1.

[5] Duarte, C. E., (2015) "Avaliação da aprendizagem escolar: como os professores estão praticando a avaliação na escola.”, HOLOS, v. 8.

[6] Falkembach, G. A. M. (2007) "Ferramentas de autoria. Universidade Federal do Rio Grande do Sul, Centro Interdisciplinar de Novas Tecnologias na Educação."

[7] Gomes, A. S., Carvalho, R, S., Melo Filho, I. J.;, Rolim, A. L. S., Monteiro, B. S. e Oliveira, G. R. S. (2009). "Amadeus: Novo Modelo de Sistema de Gestão de Aprendizagem.”, Revista Brasileira de Aprendizagem Aberta e a Distância.

[8] Leffa, V. J. (2006) "Uma ferramenta de autoria para o professor: o que é e o que faz.”, Letras de Hoje, Porto Alegre, v. 41, n. 144, páginas 189-214.

[9] Luckesi, C .C. (2005) "Avaliação da aprendizagem escolar: estudos e proposições.”, 17. ed. São Paulo: Cortez.

[10] Pimentel, M., Filippo, D. e Santoro, F. M. (2019) "Design Science Research: fazendo pesquisas científicas rigorosas atreladas ao desenvolvimento de artefatos computacionais projetados para a educação". Metodologia de Pesquisa em Informática na Educação: Concepção da Pesquisa. Porto Alegre: SBC, (Série Metodologia de Pesquisa em Informática na Educação, v. 1).

[11] Rosa, C. W., Darroz, L. M. e Marcante, T. E., (2012) "A avaliação no ensino de Física: práticas e concepções dos professores”, Rev. Electrónica Investig. En Educ. En Ciencias, vol. 7, n. 2, páginas 41-53.

[12] Santos, A. L, Luis, J. e Silva, P. G. (2008) "Formação e Práticas Pedagógicas Múltiplos Olhares no Ensino das Ciências - Artigo: A influência das Tecnologias da Informação e da [2] [2] [14] Comunicação (TIC) no Ensino das Ciências.", Recife/PE. Edições Bargaço.

[13] Vieira, V. M. O. (2002) "Portfólio: Uma proposta de avaliação como reconstrução do processo de aprendizagem.” Psicol. Esc. e Educ., vol. 6, no. 2, páginas 149-156.

[14] Ardora. (2009). Disponível em: 〈http://webardora.net>.

[15] Khan Academy. (2007). Disponível em: < http://pt.khanacademy,org $>$. 